\title{
Metabolic impact of pheochromocytoma/ paraganglioma: targeted metabolomics in patients before and after tumor removal
}

\author{
Zoran Erlic', Max Kurlbaum 2,3, Timo Deutschbein ${ }^{3}$, Svenja Nölting ${ }^{4}$, Aleksander Prejbisz ${ }^{5}$, Henri Timmers ${ }^{6}$, \\ Susan Richter ${ }^{7}$, Cornelia Prehn' ${ }^{8}$, Dirk Weismann ${ }^{3}$, Jerzy Adamski ${ }^{8,9,10,13}$, Andrzej Januszewicz ${ }^{5}$, Martin Reincke ${ }^{4}$, \\ Martin Fassnacht ${ }^{2,3,11}$, Mercedes Robledo ${ }^{12}$, Graeme Eisenhofer7, Felix Beuschlein ${ }^{1,4}$ and Matthias Kroiss ${ }^{2,3,11}$
}

${ }^{1}$ Klinik für Endokrinologie, Diabetologie und Klinische Ernährung, Universitätsspital Zürich, Zürich, Switzerland, ${ }^{2}$ Core Unit Clinical Mass Spectrometry, University Hospital Würzburg, Würzburg, Germany, ${ }^{3}$ Division of Endocrinology and Diabetology, Department of Internal Medicine I, Universitätsklinikum Würzburg, Würzburg, Germany, ${ }^{4}$ Medizinische Klinik und Poliklinik IV, Klinikum der Universität München, München, Germany, ${ }^{5}$ Department of Hypertension, Institute of Cardiology, Warsaw, Poland, ${ }^{6}$ Department of Internal Medicine, Radboud University Medical Centre, Nijmegen, The Netherlands, ${ }^{7}$ Institut für Klinische Chemie und Labormedizin, Universitätsklinikum Carl Gustav Carus, Dresden, Germany, ${ }^{8} \mathrm{Helmholtz} \mathrm{Zentrum} \mathrm{München,} \mathrm{Research} \mathrm{Unit} \mathrm{Molecular} \mathrm{Endocrinology}$ and Metabolism, Neuherberg, Germany, ${ }^{9}$ German Center for Diabetes Research (DZD), München-Neuherberg, Germany, ${ }^{10} \mathrm{Chair}$ for Experimental Genetics, Technical University of Munich, Freising-Weihenstephan, Germany, ${ }^{11}$ Comprehensive Cancer Center Mainfranken, Universität Würzburg, Würzburg, Germany, ${ }^{12}$ Hereditary Endocrine Cancer Group, Spanish National Cancer Research Centre (CNIO) and ISCIII Center for Biomedical Research on Rare Diseases (CIBERER), Madrid, Spain, and ${ }^{13}$ Department of Biochemistry, Yong Loo Lin School of Medicine, National University of Singapore, Singapore, Singapore

Correspondence should be addressed to $M$ Kroiss

Email

Kroiss_m@ukw.de

\begin{abstract}
Objective: Excess catecholamine release by pheochromocytomas and paragangliomas (PPGL) leads to characteristic clinical features and increased morbidity and mortality. The influence of PPGLs on metabolism is ill described but may impact diagnosis and management. The objective of this study was to systematically and quantitatively study PPGLinduced metabolic changes at a systems level.

Design: Targeted metabolomics by liquid chromatography-tandem mass spectrometry of plasma specimens in a clinically well-characterized prospective cohort study.

Methods: Analyses of metabolic profiles of plasma specimens from 56 prospectively enrolled and clinically wellcharacterized patients ( 23 males, 33 females) with catecholamine-producing PPGL before and after surgery, as well as measurement of 24-h urinary catecholamine using LC-MS/MS.

Results: From 127 analyzed metabolites, 15 were identified with significant changes before and after surgery: five amino acids/biogenic amines (creatinine, histidine, ornithine, sarcosine, tyrosine) and one glycerophospholipid (PCaeC34:2) with increased concentrations and six glycerophospholipids (PCaaC38:1, PCaaC42:0, PCaeC40:2, PCaeC42:5, PCaeC44:5, PCaeC44:6), two sphingomyelins (SMC24:1, SMC26:1) and hexose with decreased levels after surgery. Patients with a noradrenergic tumor phenotype had more pronounced alterations compared to those with an adrenergic tumor phenotype. Weak, but significant correlations for 8 of these 15 metabolites with total urine catecholamine levels were identified.

Conclusions: This first large prospective metabolomics analysis of PPGL patients demonstrates broad metabolic consequences of catecholamine excess. Robust impact on lipid and amino acid metabolism may contribute to increased morbidity of PPGL patients.
\end{abstract}




\section{Introduction}

Pheochromocytomas and paragangliomas (PPGLs) are rare tumors that respectively arise from chromaffin cells of the adrenal medulla and associated with sympathetic ganglia in the thorax, abdomen and pelvis. The clinical and biochemical hallmark of this tumor entity is the excessive secretion of the catecholamines epinephrine, norepinephrine and dopamine (1). In contrast, paragangliomas from parasympathetic tissue in the head and neck region are usually hormonally silent. While most PPGLs are benign, in a small proportion of PPGLs, tumor presence in organs that not usually harbor catecholamine-producing cells provides evidence of malignancy which is frequently associated with germ line susceptibility syndromes (2). Distinct molecular features of PPGL have been used for classification of the tumors that were associated with distinct catecholamine biochemical phenotypes and different clinical outcomes (3). Catecholamine excess results in a broad spectrum of clinical symptoms including the classical triad of paroxysmal headache, sweating and palpitation $(1,4)$. Hormone-dependent cardiovascular complications as well as metabolic alterations are believed to be the main drivers for PPGL-associated morbidity and mortality $(4,5$, $6,7)$. Whereas elevation of blood glucose and diabetes are frequent and well-established metabolic consequences of chronic catecholamine excess caused by PPGLs, much less is known about the broader impact on metabolism (4).

Metabolomic profiling is a relatively new strategy for the parallel and high-throughput identification and quantification of dozens to hundreds of low molecular weight molecules (metabolites) within one biological specimen. Untargeted and targeted approaches are useful, but are associated with specific advantages and disadvantages. Whereas untargeted metabolomics enable the comprehensive identification of numerous molecules without prior knowledge of their presence, there is often lack of quantitation. At variance, targeted metabolomics aims at the identification and quantitation of pre-defined molecules. By definition, a targeted metabolomics approach provides the advantage of quantification of a previously identified, but limited number of metabolites, with known chemical structures (8). The known physicochemical properties of targeted metabolites chosen for analysis also allows optimization of sample preparation, detection and quantification at relatively high sensitivity and specificity. Further advantages of targeted metabolomics include the better inter-laboratory reproducibility of assays and, thus, the potential application in consecutive studies or diagnostics (9). Accordingly, targeted metabolomics have been used to investigate numerous disorders, including profiling of patients with cardiovascular and tumor diseases $(10,11,12,13,14,15,16,17)$. Here, we applied a targeted metabolomics approach to plasma samples before and after complete surgical tumor to evaluate whether normalization of a catecholamine excess would result in changes in their metabolomic profile. Patients had been enrolled in the prospective monoamine-producing tumor (PMT) study (18). We made use of a metabolomic panel, which in the past had aided in the identification of markers for early diagnosis, assessment of prognosis or risk stratification, as well as contributed to the understanding of pathophysiological mechanisms of a variety of diseases $(10,11,12,13,14,15,16,17)$.

The aim of the current study was (i) to use this unique set of samples as a model to study the impact of chronic catecholamine excess on metabolism and (ii) to determine markers that may aid in the diagnosis of PPGL. Sampling within the PMT study was standardized to minimize random fluctuations of metabolites (18).

\section{Subjects and methods}

\section{Patients and sample collection}

Patients with confirmed PPGL who underwent surgery were from the Prospective Monoamine-producing Tumor (PMT) Study, which has been described in detail elsewhere (18). All patients had biochemical evidence of a PPGL before surgery based on plasma concentrations of normetanephrine and metanephrine above the upper cut-offs of reference intervals. In brief, upon enrollment plasma samples were obtained under standardized conditions. Blood was drawn in the morning from an indwelling cannula in the brachial vein after $30 \mathrm{~min}$ of supine rest $(10 \mathrm{~mL})$. Patients were instructed to fast overnight and to refrain from alcohol and nicotine and caffeinated beverages for $12 \mathrm{~h}$ before sampling. Blood samples collected into heparinized tubes were placed on ice or cool pads at $4^{\circ} \mathrm{C}$ before centrifugation to separate plasma. Plasma samples were stored upon centrifugation at $-80^{\circ} \mathrm{C}$ until analysis. Patients selected for the current study included a subgroup who had undergone surgery with histologically confirmed diagnosis of PPGL and availability of both preoperative and postoperative plasma samples. Postoperative samples were collected at visits scheduled at least 1 month after surgery or at the 1 and 2-year postoperative follow-up visits according to the 
PMT study protocol. Patients with metastases, defined as radiological presence of PPGL in non-chromaffin organs were excluded from the study. The PMT study protocol was approved by the University Hospital Dresden Ethics Committee (EC), the Radboud University Medical Center EC, the University Hospital Würzburg EC, the Klinikum der Ludwig-Maximilians-Universität München EC and the Institute of Cardiology EC at Warsaw. All patients provided written informed consent.

\section{Genetic screening}

A targeted gene panel (TGP) previously described was used to perform the genetic screening. This panel was designed for interrogating the presence of mutations in RET, VHL, NF1, MAX, TMEM127, SDHA, SDHB, SDHD, SDHC, SDHAF2, MDH2, FH, EPAS1, HRAS, KIF1B, MEN1, EGLN1, and EGLN2 genes (19). DNA libraries were prepared according to the manufacturer's protocol and samples were sequenced using the MiSeq platform (Illumina). Those variants classified as pathogenic, likely pathogenic or VUS (variants of unknown significance) were validated by Sanger sequencing.

\section{Laboratory measurements and targeted metabolomics}

Plasma free metanephrines for all patients before and after surgery and fractionated urine catecholamines were measured as previously described (18).

Targeted metabolomics was performed using the AbsoluteIDQ ${ }^{\mathrm{TM}}$ p180 Kit (BIOCRATES Life Sciences AG, Innsbruck, Austria) (20), which complies with the EMA 'Guideline on bioanalytical method validation' (July 21st 2011) (21) and implies proof of reproducibility within a given error range. Ten microliters of plasma from each sample were processed according to the manufacturer's recommendations. Electrospray ionization liquid chromatography-mass spectrometry (ESI-LC-MS/MS) and flow-injection (FIA) MS/MS was performed on Sciex 4500QTRAP MD (SCIEX®, Framingham, USA) MS-system coupled to an Agilent 1290 UHPLC-system (G4226A autosampler, infinityBinPump, G1316C column-oven, G1330B thermostat (Agilent, Santa Clara, USA)). Data were processed with Analyst ${ }^{\circledR}$ Software version 1.6.2 MD. LC- and MS/MS settings were set according to the manufacturer's manual (UM-P180-SCIEX-13). MetIDQ ${ }^{\mathrm{TM}}$ software (5.5.4-DB 100 Boron-2623) was used for validation and processing of MS data. For further statistics data were exported as a *.csv file. Metabolite concentrations were in $\mu \mathrm{mol} / \mathrm{L}$. To ensure comparability of data between batch measurements, each metabolite value was normalized to five human reference plasma samples included into each batch as previously described (22). Metabolites with more than $10 \%$ of concentrations below the lower limit of quantification (LLOQ) and patient samples with less than $60 \%$ of all potential analytes detectable were excluded from further analysis.

\section{Statistical analysis}

Baseline characteristics (sex, age, secretory phenotype, tumor size and localization and time interval after surgery of the postoperative blood specimen) are presented as means (normally distributed) or medians (non-normally distributed) with interval-range for numerical variables and frequencies for categorical variables. The secretory phenotype (adrenergic or noradrenergic) was determined according to plasma metanephrines measurement values before surgery as described (23). For tumor size, the maximal diameter of the largest tumor (in case of multiple) was considered. The patients with simultaneous adrenal and extra-adrenal tumors were defined as with extra-adrenal localization.

For statistical analysis, patients were stratified by sex $(24,25)$, age ( $<45$ vs $\geq 45$ years) (25), tumor localization (adrenal vs extra-adrenal), BMI ( $<25$ versus $\geq 25 \mathrm{~kg} / \mathrm{m}^{2}$ ) and secretory phenotype (adrenergic vs noradrenergic). To identify metabolic changes after surgery, we compared the preoperative and postoperative metabolite values. This analysis was restricted to those patients with confirmed post-surgical biochemical remission, defined as postoperative normalization of plasma free metanephrines. The metabolites, which resulted in significant differences in at least two different statistical methods (described later in this section), were arbitrarily defined as 'relevant metabolites'.

For baseline characteristics, comparison was performed with the Pearson chi-square for categorical variables, $t$-test for mean comparison of normally distributed data and Mann-Whitney $U$ Test for non-normally distributed numerical variables (defined according to the KolmogorovSmirnov and Shapiro-Wilk test). Correlations were tested by Spearman test and a linear regression model (after logarithmic transformation of numerical variables) was built simultaneously considering BMI, presence/absence of arterial hypertension and diabetes mellitus. SPSS (IBM Corp.), version 25 was used.

For comparison between groups, we performed a series of univariate and multivariate statistical analyses 
using the Web-based MetaboAnalyst platform (26). For these analyses, non-detectable values were replaced by the half of the minimum positive value in the original data, as per default, and a generalized logarithm transformation (GLOG) (27) was performed. For intergroup comparison we used the $t$-test/paired-t-test, fold-change analysis (paired data, not GLOG transformed), Empirical Bayesian Analysis of Microarray (EBAM), Significance Analysis of Microarray (SAM) and partial least squares discriminant analysis (PLS-DA) method. Significance was determined as $P \leq 0.05$ (two tailed). To correct for multiple testing we set the false discovery rate (FDR) $\leq 0.05$ (5\%). PLS-DA results failed significance after internal validation procedures (double cross validation and permutation testing) and was therefore not considered further for selection of metabolites (Supplementary material, see section on supplementary data given at the end of this article).

\section{Results}

\section{Patient characteristics}

In total, 56 patients (23 males and 33 females) were included in the study. A brief summary of groups of patients analyzed and the workflow applied is depicted in Fig. 1.

At baseline, there were no statistically significant clinical differences between male and female patients (Table 1). Of the entire cohort of 56 patients, 53 had complete biochemical remission after surgery, while

\section{Prospective monoamine producing tumor study (PMT)}
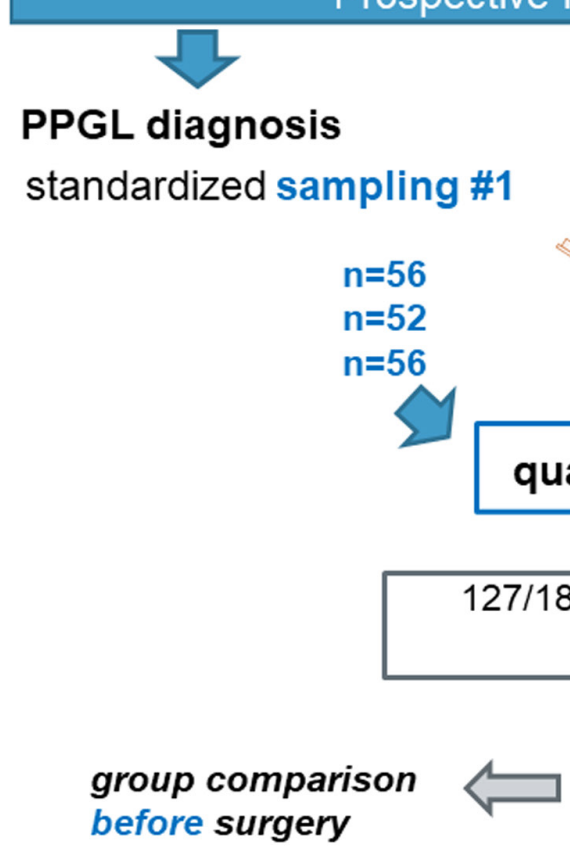

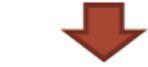

surgery

56 PPGL patients

\section{follow up}

\section{standardized sampling \#2}

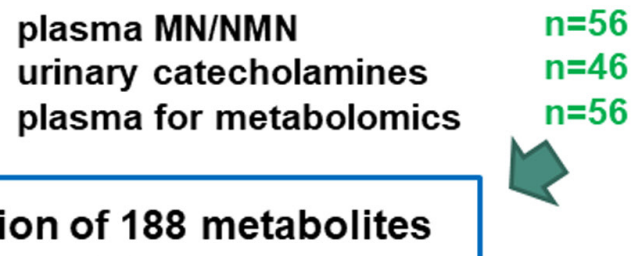

quantification of 188 metabolites

$27 / 188$ metabolites eligible for statistical analysis after data processing and selection

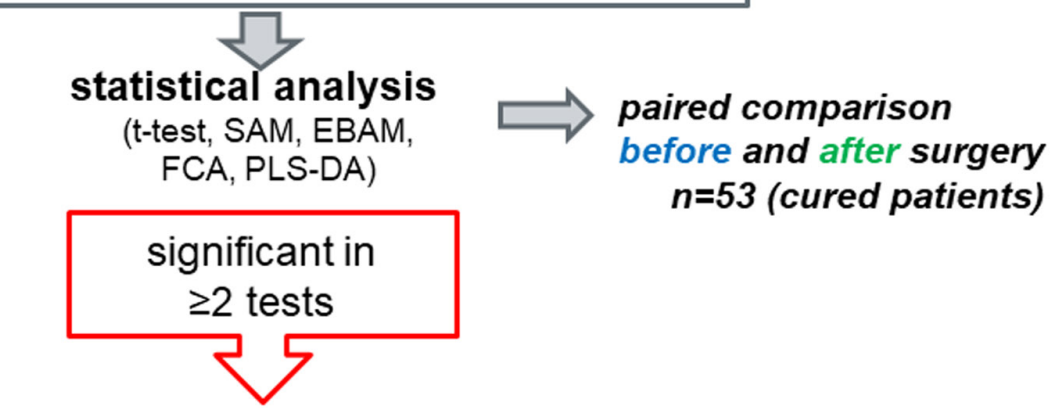

\section{«relevant metabolites»}

\section{Figure 1}

Brief summary of the methods-workflow to identify relevant metabolites. EBAM, empirical Bayesian analysis of microarray/ metabolites; FCA, fold change analysis; MN, metanephrine; NMN, normetanephrine; PLS-DA, partial least squares discriminant analysis; PPGL, pheochromocytoma/paraganglioma; SAM, significance analysis of microarray/metabolites. A full colour version of this figure is available at https://doi.org/10.1530/EJE-19-0589. 
Table 1 Patient characteristics stratified by sex. For features with missing data, number of patients with available information is specified in brackets. For numerical variables data are represented as mean for normally distributed or median for non-normally parameters with range in brackets. For categorical variables absolute and percentage value (brackets) are represented.

Antihypertensive treatment was given to 42 patients (22 patients with monotherapy, 11 patients with 2 drugs, 5 patients with 3 drugs, 3 patients with 4 drugs and 1 patient with 7 drugs). After surgery this number decreased to 21 patients (10 with monotherapy, 7 with 2 drugs, 3 with 3 drugs and 1 with 4 drugs, respectively). Percentages for antihypertensive medication are related to the total number of patients at treatment. Baseline anti-diabetic therapies were recorded in 7 out of 9 patients, of which 4 were treated with metformin (one in combination with insulin), one with sitagliptin and 2 with lifestyle measures alone. After surgery, 2 of the 4 patients with persistence of DM were treated with metformin, one with insulin and one with sitagliptin. There were no statistically significant association between preoperative metformin treatment and DM persistence as assessed by Fisher's exact test $(P=0.49)$. Two-sided $P$ value is represented.

\begin{tabular}{|c|c|c|c|}
\hline Clinical characteristics & Male $(n=23)$ & Female $(n=33)$ & $\boldsymbol{P}$ \\
\hline \multicolumn{4}{|l|}{ Preoperative features } \\
\hline Age at surgery (year) & $45.1(17-71.7)$ & $49.5(22.6-74.6)$ & 0.26 \\
\hline AHT & $18(78.3)$ & $26(78.8)$ & $1.0^{\dagger t}$ \\
\hline Antihypertensive medication, $n$ & 18 & 24 & $0.51^{t+}$ \\
\hline Alpha-blocker & $5(27.8)$ & $8(33.3)$ & \\
\hline Beta-blocker & $8(44.4)$ & $10(41.7)$ & \\
\hline ACE-inhibitor/AT1-antagonist & $10(55.6)$ & $12(50.0)$ & \\
\hline Diuretics & $5(27.8)$ & $1(4.2)$ & \\
\hline Ca channel blocker & $7(38.9)$ & $8(33.3)$ & \\
\hline Central alpha-agonist & 0 & $1(4.2)$ & \\
\hline DM & $5(21.7)$ & $4(12.1)$ & $0.46^{t+}$ \\
\hline \multirow[t]{2}{*}{ BMI $\left(\mathrm{kg} / \mathrm{m}^{2}\right)^{\dagger ; n}$} & 23 & 29 & \\
\hline & $24.8(19.5-35.3)$ & $23.6(17.2-36.9)$ & 0.32 \\
\hline \multicolumn{3}{|l|}{ Tumor localization } & $0.68^{t+}$ \\
\hline Adrenal & $20(87.0)$ & 30 (90.9) & \\
\hline Extra-adrenal & $3(13.0)$ & $3(9.1)$ & \\
\hline Tumor size $(\mathrm{cm})^{\dagger}$ & $3.9(1.8-11.0)$ & $4.6(2.0-16.0)$ & 0.17 \\
\hline \multicolumn{3}{|l|}{ Catecholamine phenotype } & 0.26 \\
\hline Adrenergic & $9(39.1)$ & $18(54.5)$ & \\
\hline Noradrenergic & 14 (60.9) & 15 (45.5) & \\
\hline \multicolumn{3}{|l|}{ Plasma metanephrine $(\mathrm{pg} / \mathrm{mL})^{\dagger}$} & 0.22 \\
\hline Metanephrine & $69(18.0-1074)$ & $157(7-1688.9)$ & \\
\hline Normetanephrine & $1156(139-3770)$ & $1067.8(85-5548)$ & 0.93 \\
\hline Urine catecholamine $(\mu \mathrm{g} / \text { day })^{\dagger ; n}$ & 21 & 31 & \\
\hline Total & $123.6(41.1-832.5)$ & $137.3(23.8-1600.9)$ & 0.70 \\
\hline Epinephrine & $9.6(2.2-538.5)$ & $24.6(0.6-226.7)$ & 0.15 \\
\hline Norepinephrine & $106.9(37.2-591.6)$ & $110.04(14.3-1576.3)$ & 0.72 \\
\hline Genetic screening, $n$ & 23 & 31 & $0.56 *$ \\
\hline Wild type & 17 & 25 & \\
\hline Germline mutation & 6 & 6 & \\
\hline RET & 1 & 2 & - \\
\hline$S D H A$ & 1 & 0 & - \\
\hline$S D H B$ & 1 & 0 & - \\
\hline$S D H D$ & 1 & 1 & - \\
\hline NF1 & 2 & 3 & - \\
\hline \multicolumn{4}{|l|}{ Postoperative features } \\
\hline Time between surgery and sampling (days) & $347.7(29-837)$ & $369.2(9-956)$ & 0.75 \\
\hline \multirow[t]{2}{*}{ Persistence of $\mathrm{AHT}$} & $n=22$ & $n=32$ & \\
\hline & $12(54.5)$ & $9(28.1)$ & 0.05 \\
\hline Antihypertensive medication & $n=12$ & $n=9$ & $0.75^{\dagger t}$ \\
\hline Alpha-blocker & $3(25.0)$ & $3(33.3)$ & \\
\hline Beta-blocker & $4(33.3)$ & $5(55.6)$ & \\
\hline ACE-inhibitor/AT1-antagonist & $8(66.7)$ & $3(33.3)$ & \\
\hline Diuretics & $1(8.3)$ & $1(11.1)$ & \\
\hline Ca channel blocker & $4(33.3)$ & $4(44.4)$ & \\
\hline \multirow[t]{2}{*}{ DM persistence } & $n=22$ & $n=31$ & \\
\hline & $4(18.2)$ & 0 & $0.03^{t+}$ \\
\hline
\end{tabular}


three patients had persistent disease with plasma free metanephrine/normetanephrine remaining elevated (one with multiple tumors and two on whom information on localization was unavailable). In addition to plasma metanephrine/normetanephrine, urine catecholamine measurements were available in 52 patients at baseline and 40 patients before and after surgery, respectively. Urinary catecholamines were used for correlation analysis with the identified "relevant metabolites" (see below). According to the patient's medical history, before surgery $44(78.6 \%)$ patients had arterial hypertension (AHT), and 9 (16.1\%) diabetes mellitus (DM). Overweight, defined as $\mathrm{BMI} \geq 25 \mathrm{~kg} / \mathrm{m}^{2}$, was present in 20 (38.5\%) patients before surgery. Following surgery, persistent AHT was documented in 21 (38.9\%), significantly more frequent in the male patients, and DM in $4(7.5 \%)$ male patients and no female patients (Table 1). Information regarding AHT and DM after surgery was not available for two and three patients, respectively. None of the included patients had a medical history of renal insufficiency. Genetic screening was performed in 54 of 56 patients and 12 (22.2\%) harbored a germline mutation. The distribution of syndromic patients (germline mutation screening positive) and non-syndromic patients (germline screening mutation negative) was not significantly different between males and females.

With respect to the 188 investigated metabolites, 115 metabolites were detectable in all samples and 127 in $\geq 90 \%$ of samples. Details on metabolites considered for each group/subgroup analysis can be found in the Supplementary section 1a (See section on Supplementary data given at the end of the article).

\section{Targeted metabolomics before surgery}

At baseline, no significant correlation between metabolite pattern and age, tumor localization, catecholamine phenotype or presence/absence of arterial hypertension was found after correction for multiple testing (Supplementary material, section 2.1b, d, f, h) (28). However, 16 metabolites were found to be significantly different between male and female patients in at least two of the performed statistical tests including 11 amino acids/biogenic amines (isoleucine, leucine, creatinine, methionine, tryptophan, tyrosine, valine, phenylalanine, glutamate, proline and histidine), carnitine, two glycerophospholipids (lysoPC acyl [a] C18:2, lysoPC a C20:3) and two sphingolipids (sphingomyelin (SM) C18:1, SM C20:2) (complete results in the Supplementary material, section 2.1a) (28). Except for the two sphingomyelins, which were more abundant in female patients, all other metabolites were more abundant in male compared to female patients. One of these metabolites (SM C18:1) was significantly correlated with preoperative total urine catecholamine $\left(r_{s}=0.342, P=0.013\right)$. One amino acid (tyrosine) significantly differ between patients with BMI $<25 \mathrm{~kg} / \mathrm{m}^{2}$ and $\geq 25 \mathrm{~kg} / \mathrm{m}^{2}$ (Supplementary material, chapter 2.1e) (4), being higher in the latter subgroup, with no significant correlation with the total urine catecholamines before surgery. Two acylcarnitines (C18:1, C18:2) and one glycerophospholipid (PC aa C40:6) were lower in the group of patients younger than 45 years with no correlation with urine total catecholamine.

\section{Changes of targeted metabolomic profiles following surgery}

Considering only the patients with biochemical remission following surgery, seven relevant metabolites were found (Fig. 2A, Table 2 and Supplementary material, section 2.2a) . Specifically, we identified two amino acids/biogenic amines (histidine, creatinine), four glycerophospholipids (phosphatidylcholine diacyl (PC aa) C42:0, phosphatidylcholine acyl-alkyl (PC ae) C42:5, $\mathrm{PC}$ ae $\mathrm{C} 44: 5$ and $\mathrm{PC}$ ae C44:6), and hexose as metabolites with significant changes following surgical normalization of catecholamine excess. Of these metabolites, histidine and creatinine levels were higher, whilst all other identified metabolites had lower values following surgery. Subgroup analyses of patients according to sex (male and female), age $<45$ years, BMI $>25 \mathrm{~kg} / \mathrm{m}^{2}$ and adrenergic catecholamine phenotype showed no statistically significant differences (Supplementary material, section 2.2b-d, g, h) . The latter might be also due to the lower number of cases in this subgroup. In contrast, in patients aged $\geq 45$ years, we identified an increase of tyrosine and PC ae C34:2 following surgery (Fig. 2D, Table 2 and Supplementary material, section 2.2i) . When analyzing the subgroup of noradrenergic tumors alone, the three metabolites creatinine, PC aa C42:0, PC ae C42:5 that could also been identified in the entire group met the criteria. In addition, seven further metabolites were found: three amino acids/biogenic amines (tyrosine, ornithine, sarcosine) with postoperative higher concentrations, two glycerophospholipids (PC aa C38:1, PC ae C40:2) and two sphingolipids (SM C24:1, SM C26:1) with lower postoperative values (Fig. 2B, Table 2 and Supplementary material, section 2.2e) . In the subgroup of patients with BMI $<25 \mathrm{~kg} / \mathrm{m}^{2}$ we confirmed the significant change of histidine following surgery. 

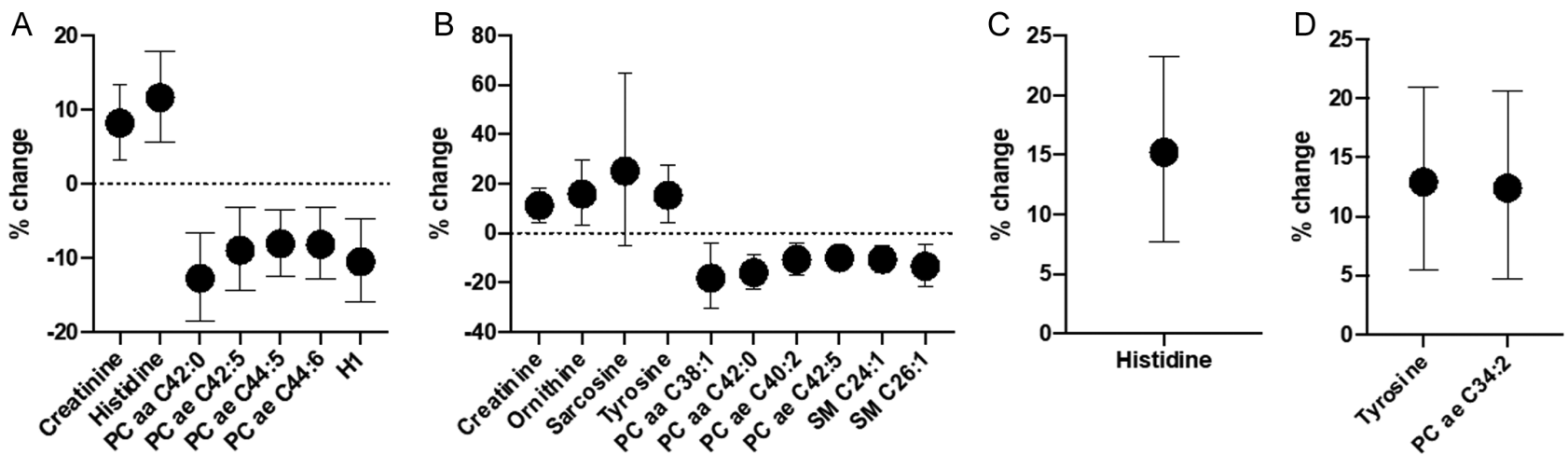

Figure 2

Metabolites defined as relevant in all (A), noradrenergic tumor phenotype (B) and subgroup of patients with $\mathrm{BMI}<25 \mathrm{~kg} / \mathrm{m}^{2}(\mathrm{C})$ and age $\geq 45$ years $(D)$, with complete postoperative biochemical remission after surgery (before-after comparison). The relevant metabolites are represented as mean of percentage (\%) change with 95\% confidence interval (CI). The mean and confidence interval were calculated after logarithmic transformation of the fold change and the results re-transformed (antilog) as percentages. The graph was designed using PRISM 8 for OS X (version 8.0.2). For metabolite name abbreviations see text and Table 2.

To test whether these differences in targeted metabolomics were related to changes in catecholamine values following surgery we performed a correlation analysis between the identified metabolites and urinary catecholamines (Table 2). From the total of 15 metabolites considered, eight displayed a weak, but significant correlation with total urine catecholamines. After including also the BMI, as well as presence or absence of arterial hypertension and diabetes mellitus in a regression model, only four metabolites (creatinine, histidine, sarcosine and hexose) were persistently associated with the urine total catecholamine values. In addition to total urine catecholamines hexoses - as expected - were linked to presence/absence of diabetes mellitus. Tyrosine was associated with BMI and sarcosine with AHT (Table 3). Further we found a significant correlation between the difference prior vs after surgery (delta) and the preoperative total urine catecholamine values for five metabolites (glutamine, histidine, PC ae C40:2, PC ae C42:5, SM C24:1 and hexose) (Fig. 3). No correlation between tumor size and extend of change (delta) could be identified.

\section{Discussion}

Here, we took advantage of a well-characterized series of PPGL patients in whom plasma sampling was performed before and after surgery in a standardized fashion. This permitted high-quality metabolomic profiling, excluding some of pre-analytic confounders such as food-dependent variations or variabilities in blood sample withdrawal and storage. By using a targeted approach with a widely targeted metabolomics technique by a liquid chromatographytandem mass spectrometry, we were able to set our result into the context of already published reports in other diseases.

Diabetes mellitus is a well-known metabolic disorder caused by PPGL (4). The finding of decrease of hexose in post-surgical specimens was hence an expected finding. More importantly, higher phospholipids PC ae C44:5, PC ae C44:6 and SM C24:1 were observed at baseline when tumor-derived catecholamine excess was present. In targeted metabolomics studies high PC ae C44:5 has been associated with decreased insulin secretion (13) and PC ae C44:6 and SM C24:1, PC ae C44:5 have been described to be higher in carriers of gene polymorphism affecting insulin secretion and sensitivity (14), which are mechanisms responsible for glucose homeostasis alteration. In line with a diabetic phenotype we found low histidine before surgery and low levels have been observed in association with insulin resistance $(15,28)$. On the other hand, in comparison to these studies, there is only a subset of phospholipids that overlaps with our results which may indicate the presence of other mechanism independent of those related to diabetes mellitus development.

Another clinical finding known in PPGL after surgery is weight gain (4). In a recent study a direct correlation between the extent of catecholamine excess and the intensity of BMI increase following surgery have been 
Table 2 'Relevant metabolites' identified from analysis of patients with complete biochemical remission comparing before and after surgery and their correlation with total urine catecholamine values. The change of the relevant metabolite value after surgery (post-OP) is represented by an upwards arrow for increased and downwards arrow for decreased postoperative concentration. The group of patients (all PPGL, noradrenergic PPGL, BMI $<25 \mathrm{~kg} / \mathrm{m} 2$, age $\geq 45$ years), in which at least two different statistical approaches found a significant change of the metabolites are marked with an " $X$ ". The rs-value represent the Spearman's correlation coefficient with positive or negative sign meaning positive or negative association of the metabolite with the catecholamine. Bold are represented the significant correlation values.

\begin{tabular}{|c|c|c|c|c|c|c|c|c|}
\hline \multirow[b]{2}{*}{ Abbrev. } & \multirow[b]{2}{*}{ Biochemical name } & \multirow[b]{2}{*}{ Post-OP } & \multicolumn{4}{|c|}{ Patient group identified } & \multicolumn{2}{|c|}{$\begin{array}{l}\text { Correlation with total } \\
\text { urine catecholamine }\end{array}$} \\
\hline & & & All & NA & $\mathrm{BMI}<25 \mathrm{~kg} / \mathrm{m}^{2}$ & $\begin{array}{c}\text { Age } \geq 45 \\
\text { years }\end{array}$ & $r_{\mathrm{s}}$ & $P$ \\
\hline \multicolumn{9}{|c|}{ Amino acids and biogenic amines } \\
\hline & Creatinine & $\uparrow$ & $x$ & $x$ & & & -0.229 & 0.03 \\
\hline & Histidine & $\uparrow$ & $x$ & & $x$ & & -0.294 & $<0.01$ \\
\hline & Ornithine & $\uparrow$ & & $x$ & & & -0.132 & 0.20 \\
\hline & Sarcosine & $\uparrow$ & & $x$ & & & -0.21 & 0.05 \\
\hline & Tyrosine & $\uparrow$ & & $x$ & & $x$ & -0.218 & 0.04 \\
\hline \multicolumn{9}{|c|}{ Glycerophospholipids } \\
\hline PC aa C38:1 & Phosphatidylcholine diacyl C38:1 & $\uparrow$ & & $x$ & & & 0.143 & 0.17 \\
\hline PC aa C42:0 & Phosphatidylcholine diacyl C42:0 & $\downarrow$ & $x$ & $x$ & & & 0.296 & $<0.01$ \\
\hline PC ae $C 34: 2$ & Phosphatidylcholine acyl-alkyl C34:2 & $\uparrow$ & & & & $x$ & 0.005 & 0.96 \\
\hline PC ae $C 40: 2$ & Phosphatidylcholine acyl-alkyl C40:2 & $\downarrow$ & & $x$ & & & 0.184 & 0.08 \\
\hline PC ae $C 42: 5$ & Phosphatidylcholine acyl-alkyl C42:5 & $\downarrow$ & $x$ & $x$ & & & 0.270 & 0.01 \\
\hline PC ae $C 44: 5$ & Phosphatidylcholine acyl-alkyl C44:5 & $\downarrow$ & $x$ & & & & 0.214 & 0.04 \\
\hline PC ae $C 44: 6$ & Phosphatidylcholine acyl-alkyl C44:6 & $\downarrow$ & $x$ & & & & 0.195 & 0.06 \\
\hline \multicolumn{9}{|l|}{ Sphingolipids } \\
\hline SM C24:1 & Sphingomyelin C24:1 & $\downarrow$ & & $x$ & & & 0.17 & 0.10 \\
\hline SM C26:1 & Sphingomyelin C26:1 & $\downarrow$ & & $x$ & & & 0.145 & 0.16 \\
\hline \multicolumn{9}{|c|}{ Monosaccharides } \\
\hline $\mathrm{H} 1$ & Hexose & $\downarrow$ & $x$ & & & & 0.422 & $<0.01$ \\
\hline
\end{tabular}

In the nomenclature of the lipids ' $C x: y$ ' indicates the lipid chain composition where ' $x$ ' is the number of carbons and ' $y$ ' the number of double bonds; a, acyl; aa, diacyl; abbrev., abbreviation; ae, acyl-alkyl; C, carbon; DOPA, dopamine; EPI, epinephrine; "n.s.", not significant; NA, noradrenergic; NOREPI, norepinephrine; PC, phosphatidylcholine; PPGL, pheochromocytoma/paraganglioma; SM, sphingomyelin.

found (29). In the current study, we found postoperative higher tyrosine levels, which in a recent homozygote twin study have been directly associated with increased BMI (16). Similarly, we found that tyrosine levels correlated with catecholamine levels in a univariate analysis, but not after considering BMI in a regression model. The underlying mechanism of tyrosine level alteration in the context of BMI change and PPGL are interesting but at the moment not clarified.

The catabolic state induced by catecholamine excess and its reversibility after surgery is impressively demonstrated by the postoperative rise in histidine and creatinine in the entire group of patients. This might in part reflect muscle wasting since muscle tissue is the main source of the circulating pool for example, of creatinine, and muscle wasting has been recently described in patients with PPGL (30).

Several of the significantly altered metabolites found in our study have been associated with the pathogenesis and/or increased risk of cardiovascular diseases. In particular, we found that histidine was lower in the preoperative sample both considering the female subgroup and the complete patient group. In the literature, low histidine levels have been described as a marker of protein energy-wasting, associated with increased inflammation and cardiovascular risk in chronic kidney failure (10) and have also been described in obese women, where an inverse correlation with inflammatory markers was reported (11). Interestingly, in PPGL patients elevated inflammatory and oxidative markers have been described in the past $(31,32,33,34)$. Therefore, one might speculate that lower preoperative histidine levels could be related or might contribute to the increased cardiovascular risk in PPGL.

The strength of the current study is the relatively large number of pre- and postoperative samples available from PPGL patients and the prospective study design in which samples were collected according to a well-controlled protocol. However the large number of metabolites in proportion to the number of studied patients and the 
Table 3 Regression analysis of the 'relevant metabolites' and total urine catecholamines including BMI and clinical presence/ absence of arterial hypertension and diabetes mellitus.

\begin{tabular}{|c|c|c|c|c|c|c|c|c|}
\hline \multirow[b]{2}{*}{ Metabolite } & \multicolumn{2}{|c|}{ Total urine catecholamines } & \multicolumn{2}{|l|}{ BMI } & \multicolumn{2}{|c|}{$\begin{array}{c}\text { Arterial hypertension } \\
\text { (absence/presence) }\end{array}$} & \multicolumn{2}{|c|}{$\begin{array}{l}\text { Diabetes mellitus } \\
\text { (absence/presence) }\end{array}$} \\
\hline & $\mathrm{B}(95 \% \mathrm{Cl})$ & $P$ & $\mathrm{~B}(95 \% \mathrm{Cl})$ & $P$ & $\mathrm{~B}(95 \% \mathrm{Cl})$ & $P$ & $\mathrm{~B}(95 \% \mathrm{Cl})$ & $P$ \\
\hline Creatinine & $-0.078(-0.123,-0.032)$ & $<0.01$ & $-0.066(-0.347,0.215)$ & 0.64 & $0.049(-0.002,0.101)$ & 0.06 & $0.057(-0.007,0.12)$ & 0.08 \\
\hline Histidine & $-0.068(-0.105,-0.032)$ & $<0.01$ & $0.002(-0.22,0.225)$ & 0.98 & $0.005(-0.036,0.045)$ & 0.82 & $0.037(-0.013,0.087)$ & 0.15 \\
\hline Sarcosine & $-0.167(-0.275,0.059)$ & $<0.01$ & $-0.353(-1.019,0.312)$ & 0.29 & $0.196(0.074,0.318)$ & $<0.01$ & $-0.082(-0.237,0.072)$ & 0.29 \\
\hline Tyrosine & $-0.040(-0.085,0.005)$ & 0.08 & $0.444(0.167,0.721)$ & $<0.01$ & $0.013(-0.038,0.064)$ & 0.61 & $-0.002(-0.065,0.060)$ & 0.94 \\
\hline Hexose & $0.054(0.025,0.083)$ & $<0.01$ & $0.176(-0.002,0.355)$ & 0.053 & $-0.013(-0.046,0.020)$ & 0.43 & $0.052(0.011,0.092)$ & 0.01 \\
\hline
\end{tabular}

Only the relevant metabolites with a significant result $(P \leq 0.05)$ are represented. For ornithine, PC aa C38:1, PC aa C42:0, PC ae C34:2, PC ae C40:2, PC ae C42:5, PC ae C44:5, PC ae C44:6, SM C24:1 and SM C26:1 no significant association could be observed in the regression model.

In the nomenclature of the lipids ' $\mathrm{Cx}: \mathrm{y}$ ' indicates the lipid chain composition where ' $\mathrm{x}$ ' is the number of carbons and " $y$ " the number of double bonds; $a$, acyl; aa, diacyl; ae, acyl-alkyl; B, unstandardized coefficient; BMI, body mass index; Cl, confidence interval; PC, phosphatidylcholine; SM, sphingomyelin.

strict selection of candidate markers might underestimate the actual metabolome-wide impact of catecholamine excess. In addition, it is conceivable that different antihypertensive drug classes might impact metabolic pathways and that the different germline mutations also may have repercussions at the metabolomic level. However, according subgroup analysis was not feasible because of the small sample size. Since the primary focus of the current study was to compare metabolome profiles before and after surgery, genotype differences were not expected to have a major impact. It is therefore prudent to suspect that the observed metabolic differences mainly represent endocrine effects of the PPGL itself. However, we cannot exclude the possibility that differences in metabolic profiles before and after surgery may have been influenced by discontinuation of antihypertensive medication after PPGL removal.

It must be emphasized that the observed alterations cannot provide causative relationships but are only observational in nature. Moreover the availability of
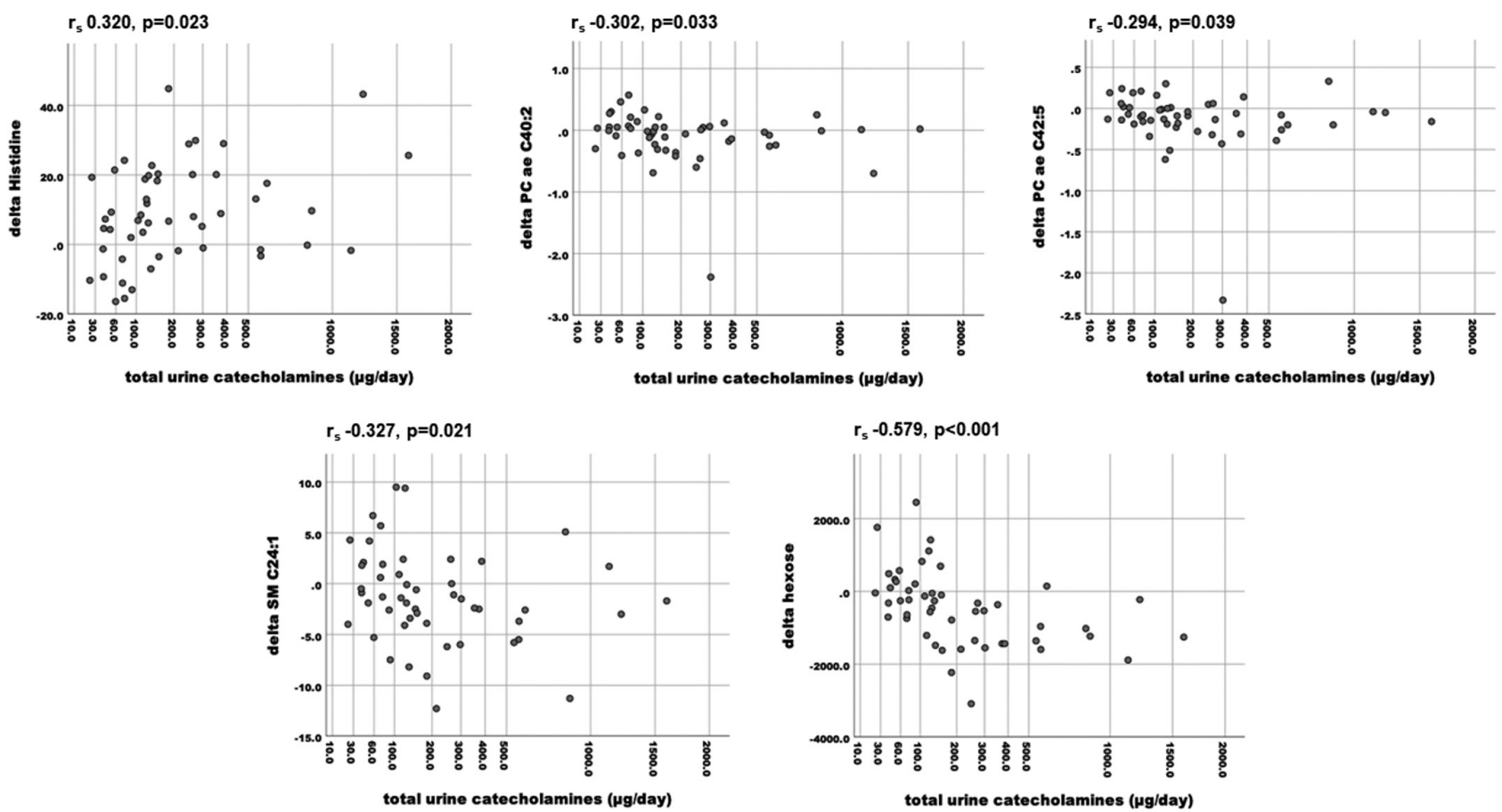

\section{Figure 3}

Simple scatter plots of the change of concentration (delta) of metabolites on the $y$-axis $(\mu \mathrm{mol} / \mathrm{L}$ ) by the total urine catecholamine values before surgery on the x-axis (exponential modified for better representation). Only the metabolites with significant correlation have been depicted. The Spearman correlation factor $\left(r_{\mathrm{s}}\right)$ and respective $P$ value are represented above each scatter-plot. 
urinary catecholamine measurements in only a subset of patients is a limitation of this study. For diagnostic purposes, the results presented here require validation in a series of PPGL patients and controls in whom PPGL has been excluded.

In summary, herein we have identified several metabolic changes, which have been associated with clinical features and cardiovascular risk factors such as diabetes and protein wasting that are also part of the clinical spectrum of PPGL patients. Norepinephrine excess is likely to be the preponderant cause of metabolic alterations. However, considering that we found only weak correlations between these metabolites and catecholamines, there might be additional upstream or downstream alterations that may not be directly related to catecholamines but rather reflect catecholamineindependent signaling for example, by other tumor-related signaling pathways such as cytokines. Further mechanistic studies explaining the observed changes would be of interest, since these mechanisms might contribute to the clinical presentation of catecholamine excess which clinically differs significantly between patients.

\section{Conclusion}

Using a targeted metabolomics approach, we could identify for the first time significant alterations in metabolic pattern in PPGL patients after successful tumor removal. Our results broaden the knowledge on the spectrum of catecholamine-induced metabolic changes which in PPGL patients however may be caused by additional endocrine features of PPGL. Observed metabolomic changes share similarities with metabolic changes associated with distinct diseases that directly relate to increased cardiovascular risk.

\section{Supplementary data}

This is linked to the online version of the paper at https://doi.org/10.1530/ EJE-19-0589.

\section{Declaration of interest}

Martin Fassnacht is on the editorial board of EJE. Martin Fassnacht was not involved in the review or editorial process for this paper, on which he/she is listed as an author. The other authors have nothing to disclose.

\section{Funding}

This study was supported by grants from the Deutsche Forschungsgemeinschaft (CRC/Transregio 205/1 'The Adrenal: Central relay of health and disease', projects S02 and B16), the European Union
Seventh Framework Programme (FP7/2007-2013) under grant agreement 259735 (ENS@T-Cancer), the European Union Horizon 2020 Program (ENS@T-HT - \#633983-2), the Instituto de Salud Carlos III (ISCIII), through the 'Acción Estratégica en Salud' (AES) (project PI17/01796), cofounded by the European Regional Development Fund (ERDF), and the Philhuman Stiftung (Vaduz, Lichtenstein).

\section{Author contribution statement}

Conception/planning of the work: Zoran Erlic, Felix Beuschlein, Matthias Kroiss, Graeme Eisenhofer; Contribution of patient samples: Timo Deutschbein, Svenja Nölting, Aleksander Prejbisz, Henri Timmers, Susan Richter, Cornelia Prehn, Dirk Weismann, Andrzej Januszewicz, Martin Reincke, Martin Fassnacht, Felix Beuschlein; Laboratory analyses: Max Kurlbaum, Matthias Kroiss, Cornelia Prehn, Jerzy Adamsky, Graeme Eisenhofer, Mercedes Robledo; Data analysis: Zoran Erlic, Max Kurlbaum, Matthias Kroiss; Interpretation of results: Zoran Erlic, Max Kurlbaum, Felix Beuschlein, Matthias Kroiss; Writing of the paper: Zoran Erlic, Max Kurlbaum, Felix Beuschlein, Matthias Kroiss. Zoran Erlic, Max Kurlbaum, Felix Beuschlein, Matthias Kroiss: These authors contributed equally to this study (shared first and senior authorship). All authors contributed to the critical interpretation of the results, reviewed the manuscript for important intellectual content, approved the final version of the manuscript, and have agreed to be accountable for his/her role in this manuscript.

\section{References}

1 Lenders JWM, Eisenhofer G, Mannelli M \& Pacak K. Phaeochromocytoma. Lancet 2005366 665-675. (https://doi. org/10.1016/S0140-6736(05)67139-5)

2 Erlic Z, Rybicki L, Peczkowska M, Golcher H, Kann PH, Brauckhoff M, Mussig K, Muresan M, Schaffler A, Reisch N et al. Clinical predictors and algorithm for the genetic diagnosis of pheochromocytoma patients. Clinical Cancer Research 200915 6378-6385. (https://doi. org/10.1158/1078-0432.CCR-09-1237)

3 Fishbein L, Leshchiner I, Walter V, Danilova L, Robertson AG, Johnson AR, Lichtenberg TM, Murray BA, Ghayee HK, Else T et al. Comprehensive molecular characterization of pheochromocytoma and paraganglioma. Cancer Cell 201731 181-193. (https://doi. org/10.1016/j.ccell.2017.01.001)

4 Erlic Z \& Beuschlein F. Metabolic alterations in patients with pheochromocytoma. Experimental and Clinical Endocrinology and Diabetes 2018127 1-8. (https://doi.org/10.1055/a-0649-0960)

5 Zelinka T, Petrak O, Turkova H, Holaj R, Strauch B, Krsek M, Vrankova AB, Musil Z, Duskova J, Kubinyi J et al. High incidence of cardiovascular complications in pheochromocytoma. Hormone and Metabolic Research 201244 379-384. (https://doi. org/10.1055/s-0032-1306294)

6 Stolk RF, Bakx C, Mulder J, Timmers HJLM \& Lenders JWM. Is the excess cardiovascular morbidity in pheochromocytoma related to blood pressure or to catecholamines? Journal of Clinical Endocrinology and Metabolism 201398 1100-1106. (https://doi.org/10.1210/jc.2012-3669)

7 Khorram-Manesh A, Ahlman H, Nilsson O, Oden A \& Jansson S. Mortality associated with pheochromocytoma in a large Swedish cohort. European Journal of Surgical Oncology 200430 556-559. (https://doi.org/10.1016/j.ejso.2004.03.006)

8 Roberts LD, Souza AL, Gerszten RE \& Clish CB. Targeted metabolomics. Current Protocols in Molecular Biology 2012 Chapter 30 Unit 3032 31-24. (https://doi.org/10.1002/0471142727.mb3002s98)

9 Siskos AP, Jain P, Romisch-Margl W, Bennett M, Achaintre D, Asad Y, Marney L, Richardson L, Koulman A, Griffin JL et al. Interlaboratory reproducibility of a targeted metabolomics platform for analysis of human serum and plasma. Analytical Chemistry 201789 656-665. (https://doi.org/10.1021/acs.analchem.6b02930) 
10 Watanabe M, Suliman ME, Qureshi AR, Garcia-Lopez E, Barany P, Heimburger O, Stenvinkel P \& Lindholm B. Consequences of low plasma histidine in chronic kidney disease patients: associations with inflammation, oxidative stress, and mortality. American Journal of Clinical Nutrition 200887 1860-1866. (https://doi.org/10.1093/ ajcn/87.6.1860)

11 Niu YC, Feng RN, Hou Y, Li K, Kang Z, Wang J, Sun CH \& Li Y. Histidine and arginine are associated with inflammation and oxidative stress in obese women. British Journal of Nutrition 2012108 57-61. (https://doi.org/10.1017/S0007114511005289)

12 Ussher JR, Elmariah S, Gerszten RE \& Dyck JR. The emerging role of metabolomics in the diagnosis and prognosis of cardiovascular disease. Journal of the American College of Cardiology 201668 2850-2870. (https://doi.org/10.1016/j.jacc.2016.09.972)

13 Floegel A, Stefan N, Yu Z, Muhlenbruch K, Drogan D, Joost HG, Fritsche A, Haring HU, Hrabe de Angelis M, Peters A et al. Identification of serum metabolites associated with risk of type 2 diabetes using a targeted metabolomic approach. Diabetes 201362 639-648. (https://doi.org/10.2337/db12-0495)

14 Then C, Wahl S, Kirchhofer A, Grallert H, Krug S, Kastenmuller G, Romisch-Margl W, Claussnitzer M, Illig T, Heier M et al. Plasma metabolomics reveal alterations of sphingo- and glycerophospholipid levels in non-diabetic carriers of the transcription factor 7-like 2 polymorphism rs7903146. PLoS ONE 20138 e78430. (https://doi. org/10.1371/journal.pone.0078430)

15 Mihalik SJ, Michaliszyn SF, de las Heras J, Bacha F, Lee S, Chace DH, DeJesus VR, Vockley J \& Arslanian SA. Metabolomic profiling of fatty acid and amino acid metabolism in youth with obesity and type 2 diabetes: evidence for enhanced mitochondrial oxidation. Diabetes Care 201235 605-611. (https://doi.org/10.2337/DC11-1577)

16 Muniandy M, Velagapudi V, Hakkarainen A, Lundbom J, Lundbom N, Rissanen A, Kaprio J, Pietilainen KH \& Ollikainen M. Plasma metabolites reveal distinct profiles associating with different metabolic risk factors in monozygotic twin pairs. International Journal of Obesity 201843 487-502. (https://doi.org/10.1038/s41366-018-0132-z)

17 Deng L, Gu H, Zhu J, Nagana Gowda GA, Djukovic D, Chiorean EG \& Raftery D. Combining NMR and LC/MS using backward variable elimination: metabolomics analysis of colorectal cancer, polyps, and healthy controls. Analytical Chemistry 201688 7975-7983. (https:// doi.org/10.1021/acs.analchem.6b00885)

18 Eisenhofer G, Prejbisz A, Peitzsch M, Pamporaki C, Masjkur J, Rogowski-Lehmann N, Langton K, Tsourdi E, Peczkowska M, Fliedner S et al. Biochemical diagnosis of chromaffin cell tumors in patients at high and low risk of disease: plasma versus urinary free or deconjugated O-methylated catecholamine metabolites. Clinical Chemistry 201864 1646-1656. (https://doi.org/10.1373/ clinchem.2018.291369)

19 Curras-Freixes M, Pineiro-Yanez E, Montero-Conde C, ApellanizRuiz M, Calsina B, Mancikova V, Remacha L, Richter S, Ercolino T, Rogowski-Lehmann $\mathrm{N}$ et al. PheoSeq: a targeted next-generation sequencing assay for pheochromocytoma and paraganglioma diagnostics. Journal of Molecular Diagnostics 201719 575-588. (https://doi.org/10.1016/j.jmoldx.2017.04.009)

20 Zukunft S, Sorgenfrei M, Prehn C, Moller G \& Adamski J. Targeted metabolomics of dried blood spot extracts. Chromatographia 201376 1295-1305. (https://doi.org/10.1007/s10337-013-2429-3)

21 Committee for Medicinal Products for Human Use (CHMP). Guideline on bioanalytical method validation. EMEA/CHMP/ EWP/192217/2009 Rev. 1 Corr. 2, 21 July 2011.
22 Haid M, Muschet C, Wahl S, Romisch-Margl W, Prehn C, Moller G \& Adamski J. Long-term stability of human plasma metabolites during storage at -80 degrees C. Journal of Proteome Research 201817 203-211. (https://doi.org/10.1021/acs.jproteome.7b00518)

23 Pamporaki C, Hamplova B, Peitzsch M, Prejbisz A, Beuschlein F, Timmers HJLM, Fassnacht M, Klink B, Lodish M, Stratakis CA et al. Characteristics of pediatric vs adult pheochromocytomas and paragangliomas. Journal of Clinical Endocrinology and Metabolism 2017 102 1122-1132. (https://doi.org/10.1210/jc.2016-3829)

24 Mittelstrass K, Ried JS, Yu Z, Krumsiek J, Gieger C, Prehn C, Roemisch-Margl W, Polonikov A, Peters A, Theis FJ et al. Discovery of sexual dimorphisms in metabolic and genetic biomarkers. PLoS Genetics 20117 e1002215. (https://doi.org/10.1371/journal. pgen.1002215)

25 Trabado S, Al-Salameh A, Croixmarie V, Masson P, Corruble E, Feve B, Colle R, Ripoll L, Walther B, Boursier-Neyret C et al. The human plasma-metabolome: reference values in 800 French healthy volunteers; impact of cholesterol, gender and age. PLoS ONE 201712 e0173615. (https://doi.org/10.1371/journal.pone.0173615)

26 Xia J \& Wishart DS. Using MetaboAnalyst 3.0 for comprehensive metabolomics data analysis. Current Protocols in Bioinformatics 2016 55 14.10.1-14.10.91. (https://doi.org/10.1002/cpbi.11)

27 Durbin BP, Hardin JS, Hawkins DM \& Rocke DM. A variancestabilizing transformation for gene-expression microarray data. Bioinformatics 200218 (Supplement 1) S105-S110. (https://doi. org/10.1093/bioinformatics/18.suppl_1.s105)

28 Feng RN, Niu YC, Sun XW, Li Q, Zhao C, Wang C, Guo FC, Sun CH $\&$ Li Y. Histidine supplementation improves insulin resistance through suppressed inflammation in obese women with the metabolic syndrome: a randomised controlled trial. Diabetologia 2013 56 985-994. (https://doi.org/10.1007/s00125-013-2839-7)

29 An Y, Reimann M, Masjkur J, Langton K, Peitzsch M, Deutschbein T, Fassnacht M, Rogowski-Lehmann N, Beuschlein F, Fliedner S et al. Adrenomedullary function, obesity and permissive influences of catecholamines on body mass in patients with chromaffin cell tumours. International Journal of Obesity 201843 263-275. (doi:10.1038/s41366-018-0054-9)

30 Lee SH, Kwak MK, Ahn SH, Kim H, Cho YY, Suh S, Song KH, Koh JM, Kim JH \& Kim BJ. Change of skeletal muscle mass in patients with pheochromocytoma. Journal of Bone and Mineral Metabolism 201937 694-702. (https://doi.org/10.1007/s00774-0180959-3)

31 Petrak O, Haluzikova D, Kavalkova P, Strauch B, Rosa J, Holaj R, Brabcova Vrankova A, Michalsky D, Haluzik M, Zelinka T et al. Changes in energy metabolism in pheochromocytoma. Journal of Clinical Endocrinology and Metabolism 201398 1651-1658. (https:// doi.org/10.1210/jc.2012-3625)

32 Bosanska L, Petrak O, Zelinka T, Mraz M, Widimsky Jr J \& Haluzik M. The effect of pheochromocytoma treatment on subclinical inflammation and endocrine function of adipose tissue. Physiological Research 200958 319-325. (https://doi.org/10.1016/S09536205(08)60153-3)

33 Turkova H, Petrak O, Skrha J, Widimsky Jr J \& Zelinka T. Pheochromocytoma and markers of oxidative stress. Physiological Research 201362 331-335.

34 Petrak O, Widimsky Jr J, Zelinka T, Kvasnicka J, Strauch B, Holaj R, Stulc T, Kvasnicka T, Bilkova J \& Skrha J. Biochemical markers of endothelial dysfunction in patients with endocrine and essential hypertension. Physiological Research 200655 597-602.
Received 29 July 2019

Revised version received 8 October 2019

Accepted 14 October 2019 